\title{
The role of community language radio for understanding creativity and wellbeing in migrant communities in Australia
}

\author{
Amanda E. Krause · Anya Lloyd-Smith · John Hajek
}

\begin{abstract}
Community radio-and community language radio specifically-occupies an important place in Australia's multicultural landscape. Members of many language communities arriving in Australia have been denied important opportunities in their home countries including outlets for self-representation and public creativity in their languages. Within Australia, radio provides an accessible means of creative expression, provides vital social connection for community members of all ages and generations, and supports social cohesion on a wider scale. This article explores how community language radio in Australia can play a critical role in supporting the wellbeing of both individuals and communities by providing an accessible and adaptable outlet for creative expression. This case study examines the practices of presenters from Australia's largest community language radio station, 3ZZZ, which reports broadcasting in around 70 languages weekly. A sample of 16 presenters from the station completed an online, mixed-methods survey. The results afford discussion of the format and composition of community language programs as a form of cultural and language maintenance, the perceived role of creativity in program design and delivery, the perceived impact of the programs for the community, and the perceived role of the program for individual and community wellbeing. The findings are considered with respect to pertinent theoretical frameworks, exploring the implications concerning creativity, community, and wellbeing. The multifaceted results we present highlight how creative community language radio participation is able to contribute positively to wellbeing in the Australian migrant context.
\end{abstract}

Keywords: community radio, ethnic radio, broadcasting, creativity, wellbeing, cultural and linguistic diversity, migration

\section{Introduction}

The processes of migration and refugee settlement have brought to Australia a diversity of languages and cultures, particularly over the last 75 years - with a significant concentration having arrived in the last decade. According to the 2016 Census, one third of the Australian population was born elsewhere, and of this group, 18 percent have arrived in the country since 2012 (Australian Bureau of Statistics, 2016). Furthermore, more than one fifth of Australians speak a language other than English in the home, and over 300 separate languages have been identified.

Settlement in a new country requires access to information about government systems and services, local news and current affairs. Successful integration is facilitated through the provision of this information in first languages, as well as through the provision of opportunities for cultural and linguistic maintenance and ongoing connections with countries of origin. Members of many recent language communities arriving in Australia have been denied important 
opportunities in their home countries including outlets for self-representation and public creativity in their languages. Alongside the national public broadcaster, SBS, which transmits radio programs in a range of community languages, an important means to achieving this for members of migrant communities is through community radio.

Community radio-and specifically community language radio-occupies an important place in Australia's multicultural landscape. Within Australia, community language radio provides an accessible means of creative expression, provides vital social connection for community members, and supports broader social cohesion (Forde et al., 2009; Kwong, 2012). Community radio serves an essential function for communities whose needs are not met by mainstream (public or private) broadcasters. In Australia, there are more than 450 community radio stations with 97 of those broadcasting in languages other than English on either a part-time or full-time basis (National Ethnic and Multicultural Broadcasters' Council, n.d.).

The largest volunteer-based community language or ethnic broadcaster ${ }^{1}$ in Australia is $3 Z Z Z$ (n.d.), being the focus of this study. Like other such stations, it stands in significant contrast to SBS, with local rather than national function, and limited financial resourcing. Its volunteer staffing, unlike SBS's professional career orientation, also provides the potential for greater individual autonomy which is of specific interest to us here. Based in Melbourne, 3ZZZ began broadcasting on a regular basis in 1989 and currently reports broadcasting in over 70 languages for over 60 ethnic groups (3ZZZ, n.d.). With such a large number of languages and cultures represented-both established communities as well as new and emerging communities - the station presents an ideal site for examining a range of approaches to programming and the impact that the programs themselves have on the wellbeing of various communities. Thus, the present work examines the experiences of $3 \mathrm{ZZZ}$ presenters in order to gain a greater understanding of how community language broadcasting can contribute to wellbeing ${ }^{2}$ by providing an accessible and adaptable outlet for creative expression.

\section{Literature Review}

An increasing body of research is highlighting the benefits of community radio, in addition to a number of studies which explore different aspects of the broadcasting of community languages in Australia (e.g., Kwong, 2012), both as it relates to the volunteer and the publicly funded (i.e., SBS) radio sectors. It is true that there are undoubtedly many challenges facing community radio, such as funding and governance (see, e.g. Order, 2016). However, given our specific interest in how wellbeing may be supported, in this section we focus on several relevant, positive themes arising from previous research on community language radio-particularly of a volunteer nature - and set out a case for current research situated in the Australian context.

\section{Maintaining and Representing Culture}

Community language radio plays a beneficial role for maintaining and representing cultures (Meadows et al., 2007; Milan, 2008; Purkarthofer, et al., 2008; van Vuuren, 2002). Community

\footnotetext{
${ }^{1}$ Since the mid-1970s, the term "ethnic" has been replaced in many domains by the more inclusive term "community languages" (e.g., "ethnic schools" are now more formally called "community language schools"), which reflects the fact that the languages are present and used within the wider Australian community, are used not only by overseas-born but also by Australian-born generations, and are learned and used by members of other ethnic groups (Clyne, 1991, p.3). The terms "ethnic radio" and "ethnic broadcasting", however, continue to be used by the sector itself, as well as by peak bodies and researchers (e.g., National Ethnic and Multicultural Broadcasters' Council, n.d.).

2 The World Health Organization's (2020, p. 1) definition of health makes reference to physical, mental, and social well-being rather than simply the absence of disease or illness. We use the term "wellbeing" rather than health, given that it transcends a strictly biomedical model of health (Rudd, 2017). Our use of the term acknowledges that people's perceived quality of life is a part of how health/wellbeing is experienced (Rudd, 2017).
} 
language broadcasting "represents a valuable cultural resource that contributes to Australia's rich and diverse cultural heritage through its commitment to maintaining, representing and reproducing local cultures" (Forde et al., 2009, p. 126). Access to the local community is particularly beneficial for emerging communities with little or no established networks (Meadows et al., 2007), as listeners also develop a "sense of belonging" where they are able to contribute to talk-back discussions on air and are even able to form relationships with the presenters (Meadows \& Foxwell, 2011, p. 100).

The use of traditional music, news and language in community language radio can assist listeners to retain a connection with their home culture and to maintain relationships with others in their community (Meadows et al., 2007; Forde et al., 2009). Community language radio is an important resource for advertising community events and local news (Forde et al., 2009). Tuning in to programs spoken in their own language also helps listeners preserve their language proficiency, as well as to teach this language to their children (Forde et al., 2009). Broadcasting traditional music has also frequently been noted as a way of maintaining ties with the country of origin and creating a sense of belonging to the new one, as well as "bridg[ing] cultural gaps between ethnic communities and mainstream Australia” (Meadows et al., 2007, p. 80).

\section{Creating a Sense of Community}

Community radio is dependent upon volunteerism for most aspects of its operation. The high level of involvement by volunteers is particular to community radio broadcasting (Milan, 2008; Purkarthofer et al., 2008; van Vuuren, 2002). Community language radio stations have been reported to average 271 volunteers per radio licensee, compared to an overall national average of 75 (Forde et al., 2009). Further, the boundaries between presenters and listeners in community radio are blurred (Meadows et al., 2007; Milan, 2008). Many listeners become producers and are in fact encouraged to do so. This interchange of roles is a defining feature of community radio and is an important distinction from mainstream radio (Meadows et al., 2007; Milan, 2008; Oliveira, 2013; Order, 2017; Purkarthofer et al., 2008).

As a result of this, community broadcasting has a "community development function" such that it can enhance social capital, being the ability people have to work together towards a common goal (Van Vuuren, 2002, p. 94). Van Vuuren (2002, p. 105) found in her research on (generalist, i.e. non-ethnic) community radio that the station with the greatest volunteer satisfaction appeared to be a part of a "dense interlocking network" of other socially aware community groups in the area. In other words, members of that community tended to volunteer their time across multiple organizations, along with experiencing a sense of belonging at the station. This function also extends from production to the audience; listeners, tuning in to hear about various social issues, in turn often become more active within the community (Milan, 2008). Community radio also disseminates content which builds community; Order (2017) found that specialized cultural programming can engage both older and younger generations in discussion, in an attempt to combat a feeling of alienation often experienced by first generation migrant youth towards their parents' home culture.

\section{Empowering Individuals and Communities}

Community radio-whether generalist or ethnic-focussed-has an empowering function, as listening to radio requires no literacy level or significant cost to access (Oliviera, 2013). As a truly accessible forum, it can lend itself to unobstructed citizen participation, which itself is a political tool of activism and social change (Meadows et al., 2007; Milan, 2008; for a different view see also Forde et al., 2002). Community radio presenters have the power to present not only their own 
ideas but also the ideas of their listeners, from under-represented communities (Milan, 2008). As producer and listener roles are blurred, listeners of community radio have more power of participation compared to those of mainstream radio and of other media.

Listeners of ethnic radio are thus given a platform which they may not have elsewhere due to their marginalized status (Milan, 2008). Van Vuuren (2006) sees community radio as being self-regulated, because the community surrounding the community radio sets the agenda. The community is then able to cover topics that may otherwise be ignored, misrepresented, or glossed over by mainstream broadcasters (Order, 2017; Purkarthofer et al., 2008).

Community radio volunteers cite a range of empowering benefits gained through their involvement, such as the acquisition of journalism training and communication skills (Purkarthofer et al., 2008), improved self-confidence and self-worth (Forde et al., 2009; van Vuuren, 2002), development of networking strategies, and numerous social and emotional benefits as outlined below (see also Order, 2017).

\section{Enhancing Wellbeing}

Community radio, including specifically community language radio, has been found to enhance the emotional, social, and physical wellbeing of its listeners (Kwong, 2012; Meadows et al., 2007; Meadows \& Foxwell, 2011; Oliveira, 2013; Order, 2017). The benefits to wellbeing have been found to be particularly relevant for marginalized communities (Meadows et al., 2007; Order, 2017). Research has shown that listening to community broadcasting can help mediate the challenges of loneliness, depression and isolation, often brought on by a traumatic migration process (Meadows \& Foxwell, 2011). The sense of being a part of a community, hearing a familiar presenter's voice, and being reminded of cultural ties by hearing news or music in the community language all contribute to an increased sense of wellbeing (Meadows \& Foxwell, 2011; Order, 2017).

Indeed, Meadows et al. (2007) and Order (2017) both name community radio as a survival tool for marginalized communities, whereas advantaged and well-established groups listen to improve their lives more generally. Meadows and Foxwell (2011) also noted that, when interviewing different types of listeners about why they listened to community radio, members of ethnic and Indigenous communities made more references to emotional and social wellbeing compared to less vulnerable groups. Moreover, Indigenous Australians believed that community radio circulated an alternative, more positive view of their own community, compared to mainstream media which could perpetuate negative views, instigating at times a feeling of shame (Forde et al., 2009; Meadows \& Foxwell, 2011).

Community radio also has the potential to improve and safeguard the health of vulnerable migrant groups, by broadcasting essential health and social services information in multiple languages. This is particularly true at times of crisis, such as the widespread bushfires in Australia during the summer of 2019-2020, and the global pandemic which emerged in 2020 (radioinfo, 2020). Additionally, community radio is also able to assist in mundane times. For example, a study by Centrelink - the Australian government body overseeing the allocation of social security payments and services-indicated that multilingual radio broadcasts were a much-utilized resource for community language groups to gain access to vital information in their language about services available to them, which many members of marginalized groups depend on for daily needs (Forde et al., 2009).

\section{Providing Opportunities for Creativity}


The flexibility of community radio allows for innovative practices. For example, creative uses of language during community radio programs can assist in engaging younger community members who may be disengaged from the community. In research conducted in the multilingual setting of Kenya, Ojwang (2015) found that a radio station that intentionally incorporated urban slang-used by young people regardless of their ethnic group - was able to effectively convey important health information to its target audience using a neutral medium.

The structure of community radio stations is typically non-hierarchical, and, to a degree, presenters have autonomy to be "creative" with the way in which they present their content (Order, 2017, p. 247; see also Milan, 2008; Purkarthofer et al., 2008). That is, community radio broadcasters can exercise autonomy in decision-making (Milan, 2008; Order, 2017; Purkarthofer et al., 2008). This autonomy includes decisions around the type of content, program format, style of delivery, and methods of audience engagement.

Although the empowering, community-building and cultural maintenance benefits of community language radio have been well-documented, the creativity aspect of community language radio, and its connection to wellbeing, have not been fully explored. Further illumination of the link between creativity and wellbeing in the community language radio context can be gained by examining a specific case study, and by drawing on the expertise of researchers across various disciplines including psychology and sociolinguistics to elucidate the results. The present research, set in the Australian context, aims to do this and will therefore deepen the understanding of how ethnic broadcasting can contribute to wellbeing by providing an accessible and adaptable outlet for creative activity and cultural expression.

\section{Aim}

We aimed to explore, from a multidisciplinary perspective and at a micro level, how ethnic community radio in Australia can enhance wellbeing by providing an accessible and adaptable outlet for creative cultural expression. Australia's largest ethnic community radio station, 3ZZZ (n.d.), was chosen as our case study.

This study was guided by the following research questions:

RQ1: What are the programming practices and motivations of different language groups in ethnic community radio?

RQ2: How does creativity feature in the practices of different language groups in ethnic community radio?

RQ3: How do these creative practices enhance individual and community wellbeing?

\section{Method}

Research Design

Following a common methodology in community radio research (e.g., Anderson, 2017; Kwong, 2012; van Vuuren, 2004), we adopted a case study approach, which allowed us to focus on the experience of volunteers at one community radio station (3ZZZ). Data were collected by means of a questionnaire with open and closed questions, as described in detail below, and again consistent with previous research in the field (e.g., van Vuuren, 2002; 2004). The mixed-method nature of the survey instrument allowed for both quantitative and qualitative data to be collected for analysis. 
Participants

Recruitment involved an email invitation circulated by $3 Z Z Z$ staff to all presenters at the station and direct, follow-up email invitations to program representatives with publicly available contact details on the station's website. Sixteen individuals filled out the questionnaire. The respondents included seven females $(43.80 \%)$ and nine males $(56.3 \%)$. Their ages ranged from 41 to $76(M=61.3, M d n=64.0)$. While the respondents currently resided in Australia, only four of the participants (25\%) were born in Australia. Participants had resided in Australia for between 4 and 71 years $(M=41.6, M d n=49.0)$. Only one participant reported that their first language was English; the first languages of the remaining individuals were French (3 participants), German (2), Greek (4), Japanese (1), Sinhala (1), Spanish (2), Turkish (1), and Yiddish (1).

The University of Melbourne approved this research (Approval number: 1955413.1). Participation was voluntary, and individuals had the opportunity to enter a prize draw for one of two \$50 AUD gift cards.

\section{Procedure and Materials}

Individuals accessed the questionnaire via a direct weblink and, after indicating their consent to participate, they completed the questionnaire as a series of webpages (hosted using Qualtrics). After completing the questionnaire, participants were thanked and debriefed.

Participants were asked to report their gender, age, country of birth, the number of years they had resided in Australia, and their first language. Individuals were asked questions about their community language program involvement. They indicated the 3ZZZ language program they were involved in, the number of years they had been involved, their role in the program, and how many hours per week they were involved in (a) preparing and (b) presenting the program on-air.

To learn about the participants' programs, they were asked: What sort of content do you include in your program?; Why do you include this content?; and, for those who indicated that they used English in their program (i.e., by responding to a yes/no question), Why do you use English in your radio program? Moreover, participants were asked whether the radio program follows the same basic format each week (yes/no), and if they felt that there is room for creativity when they are preparing or presenting their radio program (yes/no). Depending on their response, one follow up question asked: In what ways do you feel you are able to be creative when you are preparing or presenting your radio program? or Why do you think there is no room for creativity?

The next section included two open-ended questions concerning the impact of the radio programs: What do you think the role of your program is for your community (What does your program provide to your community)? and What do you think your audience gets from listening to your program? Participants responded to a third question, How important do you think it is for your community to have its own radio program?, using a five-point scale $(1=$ extremely important, $5=$ not at all important $)$. Lastly, participants responded to a set of 16 items taken from Krause, Davidson and North's (2018) 36-item scale designed to measure the benefits to wellbeing resulting from participation in music (e.g., "It allows me to be creative"; see Table 1 for all items). The items were selected as most appropriate to understanding wellbeing in the community radio context. Slight amendments were made to simplify the language for use with non-native speakers of English involved in radio broadcasting, to facilitate participant responses concerning the impact of their involvement in the radio program using a five-point scale $(1=$ disagree, $5=$ agree $)$.

Data Analysis 
A series of thematic analyses (Braun \& Clarke, 2006) were performed to identify patterns within the qualitative data. Following the process outlined by Braun and Clarke (2006), all of the responses were coded according to topic and then these codes guided the identification of themes. SPSS (version 25) was used to explore the quantitative data and calculate descriptive statistics.

\section{Results}

Program Involvement

The 16 respondents were involved in programs presented in nine different languages: Austrian, Cypriot Turkish, Esperanto, Greek, Japanese, Jewish, Mauritian, Sinhala, and Spanish. Respondents indicated that their roles included presenting $(n=13)$, production elements $(n=8)$ and language program convenor $(n=3)$; some participants $(n=9)$ reported that they had two roles. Participants had been involved with a language program at the station between 1 and 25 years, and for an average of 8.4 years $(M d n=4.5)$. Respondents stated that they would spend an average of 4 hours preparing ( $M d n=4.0$, Range: $2-8)$ and 1.1 hours ( $M d n=1.0$, Range: $0.25-2)$ presenting each week.

\section{Program Content}

A thematic analysis identified three themes pertaining to content included in their particular language program. Because individual responses included at least three, varied components, the resulting themes arose from both looking across responses as well as breaking apart individual responses.

The first theme, "entertainment", covered a range of content, including music, sports reviews, talk back segments, interviews, comedy segments, stories, travel information, historical talks, as well as topics of interest and recipes ("music, comedy and stories"). While a broad range of content were noted, some types were more prominent than others. Music was the most commonly cited type of content $(n=9)$, with responses making reference to music in general as well as specific types of musical activities ("We also promote and work with local artists often having them in as guests playing music"). In contrast, recipes and stories were only mentioned once (e.g., "food recipes"; "comedy and stories").

The second theme, "current affairs", covered topics including news, political content, and weather ("current affairs"; "science and technology in the world"; "social and political issues"). The presenters referenced covering both Australian issues ("Commentary on current affairs issues relating to Australia"; "local topics") as well as international issues from their home countries ("news from Mauritius"; "politics in Sri Lanka"). Additionally, respondents mentioned also covering social and culturally specific issues, whether local or international in scope ("workers' rights and other progressive matters"; "culturally significant issues"; "matters of social justice, the environment, war and peace").

The third theme, "culture and community events", pertained to current happenings within their local community. Though related to the current affairs theme, this type of content included announcements and information about community events and community members ("community announcements, including death notices"; "events organized by local Greek organization"; "community events"; "information from communities") and cultural activities and information ("readings from Greek literature"; "cultural activities"; "topics of interest from Cyprus and Turkey"). It was clear that presenting information about their own culture and community is important to presenters at $3 \mathrm{ZZZ}$, making it a unique, tailored program for listeners. 


\section{English Content}

Half of the programs (7 out of 14) included content in English. A thematic analysis of the reasons for including English content revealed two themes: (1) the lack of translatability of certain (Australian) English terms or concepts, as well as (2) a need to accommodate differing levels of community language proficiency among presenters and listeners. With regard to the use of English vocabulary or translations, participants indicated that it was easier as a speaker to use familiar terms ("sometimes it's because we haven't got a better word or expression to convey the message") as well as indicating a desire to convey the intended meaning clearly to their audience ("for clarification of terms to Australian-born listeners"). Participants also indicated a desire to accommodate the language skills of both the presenters ("my co-presenter feels as though she has limited knowledge of the Greek language so she presents primarily in English") and guests ("we also sometimes conduct interviews in English when the interviewee is someone who was born and educated here"). Including English content is an inclusive practice relative to the program's audience and larger Australian context. In addition to catering to multiple generations (for whom language skills and preferences vary), presenters recognized that using English helps to "reach out to Australian members of the community" and attract Australian-born listeners.

\section{Motivations for Content Inclusion}

There was a complexity to the varied responses to the question "why do you include this content?". Two themes were identified: "of interest" (with three sub-themes) and "platform for the community".

The "of interest" theme was divided into "of interest to the listeners", "diversity", and "of interest to presenters". Several presenters mentioned they included their particular content because it was what their listeners want ("it is of interest to the listeners"; "this is what our listeners are interested in") or had been requested ("feedback from listeners helps to inform the content"; "most requested"), indicating that listener input helps to shape the type of content presented. Diversity was expressed as including chosen content to cater to the diversity of their listening audience ("variety to cater to the diversity of the audience") as well as "to expand the parameters of understanding of listeners/viewers". In addition to considering the listening audience, several $3 Z Z Z$ presenters stated they include certain content because it is of interest to them ("it is what I am interested in"), suggesting presenters consider their own interests as well.

With respect to the "platform for the community" theme, respondents indicated that their programs provide an opportunity for the community to share their events and find out what is happening locally ("to help community organizations"; "share their talent with the community"). Additionally, the radio program provides a way for the community to celebrate their members' achievements ("we like to share and celebrate their achievements") maintain culture and language ("provide a program for Greek language listeners"; "no other program covered these topics with enough detail").

\section{The Role of Creativity}

While $84.6 \%$ of the participants indicated that the program's format was consistent each week, all respondents indicated that there was room for creativity in their program involvement. A single theme concerning "autonomy" was identified in terms of how the presenters spoke about being creative when preparing or presenting their radio program. While several participants broadly expressed that being creative when preparing or presenting their radio programs is due to having autonomy with regard to the program content ("we have autonomy as programmers"; "no restrictions on content"), two sub-themes further delineated that autonomy facilitated 
presenter creativity with regard to (1) the style of presentation/communication and (2) presenting community-specific content.

While how the content is presented ("having sections that actually bring something new or interesting to listeners") hints at the type of content included, this theme is more concerned with how the program is presented. Thus, it includes the order within which they present the program content ("choosing the order of the news or the style in what I want to communicate and choosing the music as well"). In this way, even though the program might use a consistent format, this kind of autonomy facilitates presenters' creativity: they choose their own ways of communicating and presenting as they best see fit for their listeners.

Participants' responses suggest that creativity as autonomy was also expressed in terms of what content is presented, indicating the importance of flexibility with regard to content choices. Community-specific content appeared to fall into three broad types: events and topics, guest interviews, and the type of music chosen for the program. Radio presenters were able to focus on certain issues or happenings in the world and of interest to their community. For example, "when there are special programs (e.g., Mothers' day)" or special cultural holidays like Mauritius Independence Day or Divali, they are able to tailor their radio program to that event and play special music or even prepare a quiz. In this way, presenters can develop programming around what is currently important in their community ("the focus each week can be different depending on what is happening in the community"). This notion of creativity through autonomy and flexibility extends to inviting "guests according to topical issues and events" as presenters indicated that the people they invite on their program and the topics they discuss help them to be creative. Additionally, being able to choose the style of music they want to present ("exploring different types of Greek music"; "choose a certain singer or a certain type of music"), allows the presenters to be creative.

\section{Program Impact}

All participants believed that it was highly important for their community to have its own radio program. On a scale where 1 was rated as extremely important, the mean response was $1.3(\mathrm{Mdn}=$ 1.0). Two higher-order themes were identified from a thematic analysis concerning the perceived role of the radio program for the community: a sense of belonging/connection (with three subthemes: connection to language, culture, and the community) and information and entertainment.

The first sense of belonging sub-theme, "connection to language", was predominant: most presenters spoke about the important role of their radio program in helping their listeners stay connected with their languages ("familiarity in language"; "contact with the Greek language and culture"; "hearing their language spoken"; "connection with their own language").

The second sub-theme, "connection to culture/cultural identity", related to how program listeners are able to stay connected to what is happening within their culture ("cultural memories"; "it provides them a door to new and fresh culture produced in their own language"). $3 Z Z Z$ programming provides an opportunity for listeners who have moved to Australia to engage with, and remained connected to, their original culture ("cultural identity"; "cultural memories and songs of their countries") and also introduces broader community members to new cultures ("they get to know the local Hispanoamerican culture"; "keeps listeners informed about the Esperanto movement, as well as informing our international listeners"). In this way, radio programs offer opportunities to express and enact cultural identity.

The third sub-theme "connection to community" refers to how their programs function as an information board for their communities. Presenters' radio programs offer listeners a way to 
know what is happening within their community and allows them to stay connected ("the community gets information"; "an excellent way to communicate with the community"; "community events").

The second theme "information and entertainment" pertains to each of the two topics separately. This sub-theme of information does overlap with the "connection with community" sub-theme, as sometimes the information is community-specific. For instance, presenters noted that their radio program provides a platform for their listeners to receive news and information about what is happening in their community and the world around them ("unique in-depth news and analysis"; "news from the islands we come from"; "keeps listeners in Australia informed"). The focus of the radio program was also reported as being to entertain listeners ("entertainment"; "fun and entertainment"; "entertainment and new trends in music"), such that "enjoyment" was also stated as a listener outcome. Importantly, given the nature of the programming, 3ZZZ listeners can enjoy unique content that is different from mainstream media ("it provides diversity of information"; "the community gets information it wouldn't get in mainstream media"; "unique in-depth news and analysis of all sorts of issues").

\section{Wellbeing}

Participants reflected on the wellbeing benefits of their participation via a short, quantitative measure (sample means and standard deviations are presented in Table 1). While the small sample size precluded additional analyses, the widespread high level of agreement with the items indicates positive associations between the participants' involvement with community language radio and their own, personal wellbeing. The perceived benefits to wellbeing were related to the different domains of wellbeing -including social, emotional, personal growth, and quality of life. Moreover, the creativity item was the most highly endorsed item, suggesting that the presenters appreciated the creative aspect of their work. 
Table 1.

Descriptive statistics concerning the wellbeing items $(N=14)$

\begin{tabular}{llll}
\hline Item & $M$ & $M d n$ & $S D$ \\
\hline It allows me to be creative & 4.7 & 5.0 & 0.5 \\
It makes me feel more connected to other people who speak & 4.6 & 5.0 & 0.6 \\
my language in Melbourne & & & \\
It gives me a sense of belonging & 4.6 & 5.0 & 0.8 \\
It helps me develop friendships & 4.6 & 5.0 & 0.8 \\
It helps me learn new skills & 4.6 & 5.0 & 0.7 \\
It makes me feel more connected to other people who speak & 4.4 & 5.0 & 0.8 \\
my language around the world & & & \\
It helps me bond with other people & 4.4 & 5.0 & 0.8 \\
It makes me feel uplifted & 4.4 & 5.0 & 0.8 \\
It helps me improve myself & 4.4 & 5.0 & 0.8 \\
It improves my mood & 4.3 & 4.0 & 0.7 \\
It helps me feel more positive about life & 4.3 & 5.0 & 0.9 \\
It makes me feel more connected to the wider Australian & 4.2 & 4.0 & 0.9 \\
community & & & \\
It is important to my sense of identity & 4.2 & 4.5 & 1.1 \\
It helps me learn about myself & 4.1 & 4.5 & 1.0 \\
It helps me to think about who I am & 3.9 & 4.0 & 1.2 \\
It increases my self-esteem & 3.9 & 4.0 & 1.2 \\
\hline
\end{tabular}

Note. Responses were made using a 5-point scale, where $1=$ disagree, and $5=$ agree.

\section{Discussion}

In this study, we aimed to explore the programming practices of different language groups to consider how ethnic community radio might (a) provide a platform for creative practice and (b) influence wellbeing. Each of these elements are discussed in turn below. Three interconnected themes dominated the thematic analysis: language, culture and community. These three elements are central to the $3 \mathrm{ZZZ}$ presenters' involvement as well as the program's impact on themselves, their listening audience, and their community.

\section{Programming Practices and Motivations}

It is difficult to treat these themes of language, culture and community separately when considering programming practices and motivations (RQ1). As $3 Z Z Z$ is an ethnic community radio station, programs are linked to a specific ethnic or language community and each individual community drives content decisions and shapes the program. The representation, promotion, and maintenance of language, culture and community are facilitated through programming content that is information- and entertainment-focused. The languages and cultures in question are given a forefront position, such that the community and listening audience are central to programming decisions and presenter practices, resulting in $3 \mathrm{ZZZ}$ providing a platform for bringing the community together and situating the language community with the broader multicultural local community.

It is clear that $3 \mathrm{ZZZ}$ provides a platform for media representation for both presenters and listeners to connect to their (local and home) culture and communities, their language and their identity. In doing so, 3ZZZ programming helps people feel a sense of belonging. One presenter stated they wished "to be a serious and credible 'bridge' between Australia, Greece, and Greek- 
Australia". The metaphor of a bridge is particularly apt in describing the presenters' goals and practices - there is a clear desire to draw together people from different generations, as well as to appeal to migrant and native-born listeners, and local and international listeners.

\section{Creativity}

Importantly, participants' responses drew attention to the creative aspects of participation in community language radio. When asked about being creative, the presenters placed the emphasis on how the content is produced, demonstrating the importance of considering creativity in terms of process rather than product. Thus, with regard to RQ2 - which asked how creativity features in the practices of different language groups in community radio-creativity can be understood here using a process-based lens (Hogan et al., 2018). Understanding creativity in community language broadcasting means not simply referring to a trait that a person might have or the end products that are new or innovative, but rather the acts that people do; this is in line with Bernard's (2012) argument that the concept of creativity must be broadened.

From a stricter, genius ("Big C") perspective on creativity, the presenters' behaviors may not be deemed creative; however, the presenters' actions suggest mundane, everyday ("little $c^{\prime \prime}$ ) creativity ${ }^{3}$. In aiming to cater to a diverse listening audience, presenters expressed their motives to develop content of interest and importance to their community. For instance, the process of navigating language (via intentionally using English) to promote inclusion and to accommodate the language proficiency and language preferences of fellow presenters and listeners could indeed be considered as creative. From the responses, it was apparent that the freedom and flexibility of the community radio format (Milan, 2008) affords the presenters the opportunity to be creative in how they construct, produce, and perform their programming. In the present study, we have interpreted this as autonomy, and previous research has suggested that the level of autonomy community radio station presenters have in making decisions about their programs can be empowering (Milan, 2008). Thus, we argue that such autonomy might be an antecedent for creativity in this radio context. However, we do recognize that simply having high levels of autonomy or freedom may not necessarily equate to high levels of creativity. Indeed, it is interesting to consider whether the presenters themselves (or the listening audience) consider their involvement as creative. For instance, the presenters' sustained involvement in creating radio content might alter their opinion as to whether or not the process or resulting products are deemed creative. To the presenters, it may simply be the result of their hard work, but to an outsider, such effort and products may be labelled as "little c" creative.

\section{Wellbeing}

We were also interested in considering how creativity may be linked to wellbeing in ethnic community radio (RQ3). Creativity is recognized as being essential for survival and prosperity (Küpers et al., 2018), as well as for positive psychological development (Karwowski et al., 2017) and bolstering health and wellbeing (Silvia et al., 2014). Our findings suggest that surveyed presenters believe their involvement in community radio affords them, amongst other things, the opportunity to be creative, which in turn has consequences for their wellbeing.

\footnotetext{
${ }^{3}$ While much creativity research has historically been concerned with genius or exceptional individuals (so-called "Big C creativity"), it is important to consider everyday creativity ("little c creativity"), which refers to daily creative actions by ordinary people (Ivcevic, 2007; Silvia et al., 2014; Tanggaard, 2015). Everyday creativity has been defined in terms of originality displayed during work and leisure activities throughout daily life: it is about what and how things are done (Richards, 2010). Because typical acts of everyday creativity can include self-expression in daily pursuits (Ivcevic, 2007) and because everyday creativity is not only about a product but also about the process (Richards, 2010; Hogan et al., 2018), it follows that we can consider the presenters' actions from this framework.
} 
The wellbeing experienced by those involved in community language radio can be examined through the lens of research in the closely related field of music psychology. Ruud (2017, p. 589) theorized that music can act as a "cultural immunogen" because it "protects, promotes, and maintains our health and quality of life". Ruud's view states that music engagement contributes to four aspects of wellbeing: a sense of vitality, a sense of agency, a sense of belonging, and a sense of coherence and meaning (Ruud, 2017). Though Ruud's focus was music, we can extend this framework to consider ethnic radio's role in maintaining and promoting wellbeing and quality of life. Indeed, the present findings suggest that ethnic community radio promotes Ruud's four aspects of vitality, agency, belonging, and coherence/meaning.

For the presenters themselves, their participation in ethnic community broadcasting appears to promote all four aspects. The content motives demonstrate how the focus on their language and community gives presenters a sense of meaning. Further, as representatives of the station and their language group, the presenters are able to feel a sense of belonging. Their level of autonomy not only promotes flexibility and creativity, but it gives presenters a sense of empowerment or agency. By volunteering, these presenters are taking an active role in their communities, which likely promotes a sense of vitality.

It is particularly interesting to consider how ethnic community radio also promotes wellbeing beyond that of the presenters - that of the programs' listeners and language communities. The presenters are not only recipients, but they are also conduits - helping to promote the wellbeing of others, and their communities. This is most clearly evident with regard to Ruud's sense of belonging. The presenters' focus on creating a community platform with their programming provides a way for listeners to connect, stay informed (of current affairs and local events), and actively participate in their communities. This helps to create a sense of belonging for those living in the community. At the same time, the language programs create the opportunity for listeners to connect to their language, culture and heritage. Again, this would help create a sense of belonging because highlighting and including their culture assists them to move beyond their marginalized status (Milan, 2008). Additionally, this recognition and promotion of maintaining language and culture assists in creating a sense of meaning, and helps these communities thrive. Thus, we argue that, like music, ethnic community radio functions as a cultural immunogen.

More broadly, the findings concerning wellbeing can be considered from the framework of Self-Determination Theory, and the role of basic psychological needs in particular (Ryan \& Deci, 2002). According to Self-Determination Theory, the needs of competence, relatedness, and autonomy influence people's experiences of personal growth, vitality, and wellbeing (Lombas \& Esteban, 2018; Milyavskaya \& Koestner, 2011). Autonomy is defined as feeling that one's actions are self-governed and self-endorsed; relatedness concerns feeling connected socially; and competence refers to feeling that one's efforts are effective (Deci \& Ryan, 2000).

The community radio sector enables the acquisition of broadcasting and communication skills (Purkarthofer et al., 2008) which can facilitate a sense of competence; research also indicates that psychological needs are fostered in autonomy-supportive environments (e.g., Oostlander et al., 2014). It is apparent that $3 Z Z Z$ presenters have control over their program content and format, the skills to produce and broadcast programs on a weekly basis, and an understanding of the role of the programs for their community, such that we infer their autonomy and competence are well supported in their broadcasting pursuits. Thus, not only do autonomy and competence facilitate creativity, but they also promote perceived individual wellbeing.

The promotion of relatedness is certainly clear given the consideration, and role, of the community in ethnic community broadcasting. Indeed, community is both a motivation and an outcome in this context. There is an inherent sociability to community radio (Vidal, 2019), and 
the presenters would experience membership of multiple, intersecting communities (e.g., their broadcasting group, their language community and the broader listening community). Given the fact that social contexts that support the three basic psychological needs promote engagement and positive outcomes (Deci \& Ryan, 2008), leveraging the promotion of the need for relatedness likely leads to higher levels of engagement.

There is a nice synergy in the fact that satisfying the need for relatedness facilitates both the presenters' wellbeing and has benefits for and within the community. In this way, the benefits are not unidirectional. It is clear that relatedness bolsters social capital, which, in turn, bolsters wellbeing. As Ruud (2017, p. 596) stated:

Our belongingness, i.e. our relationships with other people will likewise always be an important factor in defining our state of health, which depends to a significant extent upon our "social capital"-that is, how well we are integrated with or at least connected to the community. According to Robert Putnam, many studies have established beyond reasonable doubt that social connectedness is one of the most decisive determinants of our wellbeing (Putnam, 2000, p. 326).

Indeed, there is additional evidence that social capital positively influences migrants' quality of life (Adedeji, 2019; see also Ehsan et al., 2019).

\section{Limitations and Future Research}

The present study points to a number of directions for further research. Firstly, this study was limited by a small sample size and single case study design. A larger sample size across multiple community radio stations around Australia would enable a broader examination of trends and facilitate a focus on new and emerging community languages not represented in this case study. Secondly, the findings suggest that Self-Determination Theory could provide a useful framework for designing a larger-scale, quantitative study that would measure basic psychological needs, wellbeing (necessarily adapted for use in the more complex, migrant context) and social capital. Additionally, interviews and focus groups would also be helpful in eliciting a more nuanced understanding of creativity and wellbeing in the community radio sector.

Thirdly, it is important that future research include listeners' perspectives to further understand how presenters are able to influence listener and community wellbeing. For instance, research could examine how the interaction between presenters and listeners (such as during talkback segments) enables listeners to feel a part of the creative process, and enhances their sense of competence, relatedness, and autonomy - thereby promoting their wellbeing.

Finally, for the presenters who participated in this research, creativity existed within the process of creating and delivering programming, rather than through a focus on judging the product. Additional work is needed to further delineate creativity in community language radio; one interesting avenue may be to consider an emerging stream of creativity research that focuses on innovative work around solving problems that communities and societies face (Amabile, 2017, p. 336). It may be fruitful to examine the types of problem-solving practices that are utilized by community language groups when broadcasting during community events and emergencies (e.g., health pandemics, bushfires, floods), and to frame this through the lens of creativity. In line with Glăveanu's (2020) argument that movement and mobility creates possibilities for creativity and innovation, another avenue may be to compare programming practices of new and emerging migrant communities with more established communities as well as new radio programs and stations with their more-established counterparts. 


\section{Conclusion}

Using $3 Z Z Z$ as a case radio station to examine community language radio, this research has explored how community language radio in Australia can enhance the wellbeing of its practitioners by providing an accessible and adaptable outlet for creative expression. The findings highlight how the community broadcasting context allows presenters to have their needs of autonomy, relatedness and competence met, and how the process of preparing and presenting community language radio programming is creative in nature. These presenters' practices are largely driven by explicit considerations of their listening communities and their practices are perceived to lead to wellbeing benefits not only for the presenters but also for the listeners and their communities. It is hoped that these findings will contribute to the body of research on the importance of the community radio sector in the Australian context and beyond. While much remains to be explored and understood, these findings indicate that the ethnic community broadcasting sector appears on many levels to be a mechanism for enhancing migrant wellbeing in Australia.

\section{Acknowledgements}

This research was conducted at The University of Melbourne with the support of a Creativity and Wellbeing Research Initiative (CAWRI) Seed Funding 2019 grant and a Faculty of Arts' 2019 Inter-Faculty Incubator Research Project grant. The authors wish to acknowledge Jessica Phillips, Dina Saheb, and Ambrin Hasnain for their contributions as research assistants and express sincere gratitude to all of the participants who have participated in this research.

\section{Conflict of interest statement}

The authors report no conflicts of interest.

\section{Authors}

Amanda E. Krause

James Cook University \& The University of Melbourne

Anya Lloyd-Smith

The University of Melbourne

John Hajek

The University of Melbourne

\section{Publishing Timeline}

Received 2 September 2020

Accepted 26 November 2020

Published 17 December 2020

\section{References}

Adedeji, A. (2019). Social capital and migrants' quality of life: A systematic narrative review. Journal of International Migration and Integration. Advance online publication. https://doi.org/10.1007/s12134$\underline{019-00724-6}$

Amabile, T. M. (2017). In pursuit of everyday creativity. The Journal of Creative Behavior, 51(4), 335-337. https://doi.org/10.1002/jocb. 200

Anderson, H. (2017). The institutionalization of community radio as a social movement organization: 4ZZZ as a radical case study. Journal of Radio \& Audio Media, 24(2), 251-269. 
Australian Bureau of Statistics. (2016). Cultural Diversity in Australia 2016 Census Data Summary. Australian Bureau of Statistics.

Bernard, P. (2012). Musical creativities in real world practice. Oxford University Press.

Clyne, M. (1991). Community languages: The Australian experience. Cambridge University Press.

Deci, E. L., \& Ryan, R. M. (2000). The "what" and "why" of goal pursuits: Human needs and the selfdetermination of behavior. Psychological Inquiry, 11(4), 227-268.

Deci, E. L., \& Ryan, R. M. (2008). Facilitating optimal motivation and psychological well-being across life's domains. Canadian Psychology, 49(1), 14-23. https://doi.org/10.1037/0708-5591.49.1.14

Ehsan, A., Klaas, H. S., Bastianen, A., \& Spini, D. (2019). Social capital and health: A systematic review of systematic reviews. SSM-Population Health, 8, 100425. https://doi.org/10.1016/j.ssmph.2019.100425

Forde, S., Meadows, M., \& Foxwell, K. (2002). Community radio, radicalism and the grassroots: Discussing the politics of contemporary Australian community. Transformations, 4, 1-9.

Forde, S., Foxwell, L., \& Meadows, M. (2009). Developing dialogues: Indigenous and ethnic community broadcasting in Australia. Intellect Books.

Glăveanu, V. (2020). Mobilities and human possibility. Palgrave MacMillan. https://doi.org/10.1007/978-3$\underline{030-52082-3}$

Hogan, J., Murdock, K., Hamill, M., Lanzara, A., \& Winner, E. (2018). Looking at the process: Examining creative and artistic thinking in fashion designers on a reality television show. Frontiers in Psychology, 9: 2008. https://doi.org/10.3389/fpsyg.2018.02008

Ivcevic, Z. (2007). Artistic and everyday creativity: An act-frequency approach. The Journal of Creative Behavior, 41(4), 271-290. https://doi.org/10.1002/j.2162-6057.2007.tb01074.x

Karwowski, M., Lebuda, I., Szumski, G., \& Firkowska-Mankiewicz, A. (2017). From moment-to-moment to day-to-day: Experience sampling and diary investigations in adults' everyday creativity. Psychology of Aesthetics, Creativity, and the Arts, 11(3), 309-324. http://dx.doi.org/10.1037/aca0000127

Küpers, E., Van Dijk, M., \& Lehmann-Wermser, A. (2018). Creativity in the here and now: A generic, micro-developmental measure of creativity. Frontiers in Psychology, 9: 2095. http://dx.doi.org/10.3389/fpsyg.2018.02095

Krause, A. E., Davidson, J. W., \& North, A. C. (2018). Musical activity and well-being: A new quantitative measurement instrument. Music Perception, 35(4), 454-474. https://doi.org/10.1525/MP/2018.35.4.454

Kwong, M. (2012). Australia's community radio: Connecting Asian youths to the homeland and beyond. 3CMedi, 7. https://www.cbaa.org.au/3c-media/issue-7-\%E2\%80\%93-december-2012

Lombas, A. S., \& Esteban, M. Á. (2018). The confounding role of basic needs satisfaction between selfdetermined motivation and well-being. Journal of Happiness Studies, 19, 1305-1327. https://doi.org/10.1007/s10902-017-9874-x

Meadows, M., \& Foxwell, K. (2011). Community broadcasting and mental health: The role of local radio and television in enhancing emotional and social well-being. The Radio Journal - International Studies in Broadcast and Audio Media, 9(2), 89-106.

Meadows, M., Forde, S., Ewart, J. \& Foxwell, K. (2007). Community media matters: An audience study of the Australian community broadcasting sector. Griffith University. http://hdl.handle.net/10072/396240

Milan, S. (2008). What makes you happy? Insights into feelings and muses of community radio practitioners. Westminster Papers in Communication and Culture, 1, 25-43.

Milyavskaya, M., \& Koestner, R. (2011). Psychological needs, motivation, and well-being: A test of selfdetermination theory across multiple domains. Personality and Individual Differences, 50, 387-391. https://doi.org/10.1016/j.paid.2010.10.029

National Ethnic and Multicultural Broadcasters' Council. (n.d.). Australian Ethnic Community Broadcasting [fact sheet]. https://www.nembc.org.au/

Ojwang, B. O. (2015). Language, creativity and radio: Encoding urban youth identity in Kenya. The Journal of Pan African Studies, 7(8), 121-139.

Oliveira, M. (2013). Sounds and identity: The role of radio in community building. In G. Stachyra (Ed.), Radio. Community, challenges, aesthetics (pp. 177-188). Maria Curie-Sklodowska University Press. 
Oostlander, J., Güntert, S. T., \& Wehner, T. (2014). Linking autonomy-supportive leadership to volunteer satisfaction: A self-determination theory perspective. Voluntas, 25, 1368-1387. https://doi.org/10.1007/s11266-013-9395-0

Order, S. (2016). Australian community radio: Funding challenges and dilemmas. 3CMedia: Journal of Citizen's Community and Third Sector Media, 8, 61-75. https://www.cbaa.org.au/issue-8-\%E2\%80\%93april-2016

Order, S. (2017). All the lonely people, where do they all belong: Community radio and social connection. Radio Journal: International Studies in Broadcast \& Audio Media, 15(2), 243-258. https://doi.org/10.1386/rjao.15.2.243 1

Purkarthofer, J., Pfisterer, P., \& Busch, B. (2008). 10 years of community radio in Austria: An explorative study of open access, pluralism and social cohesion. Austrian Regulatory Authority for Broadcasting and Telecommunications.

Putnam, R. D. (2000). Bowling alone. The collapse and revival of American community. Simon and Schuster. radioinfo. (2020, May 5). NEMBC starts national news service for multicultural communities during the COVID-19 crisis. https://www.radioinfo.com.au/news/nembc-starts-national-news-servicemulticultural-communities-during-covid-19-crisis

Richards, R. (2010). Everyday creativity: Process and way of life - four key issues. In J.C. Kaufman, \& R. J. Sternberg (Eds.), The Cambridge handbook of creativity (pp. 189-215). Cambridge University Press.

Ruud, E. (2017). Music, identity, health. In R. A. R. MacDonald, D. J. Hargreaves, \& D. Miell (Eds.), Handbook of musical identities (pp. 589-601). Oxford University Press. https://doi.org/10.1093/acprof:oso/9780199679485.001.0001

Ryan, R. M., \& Deci, E. L. (2002). An overview of Self-determination Theory: An organismic-dialectical perspective. In E. L. Deci \& R. M. Ryan (Eds.), Handbook of self-determination research (pp. 3-36). The University of Rochester Press.

Silvia, P. J., Beaty, R. E., Nusbaum, E. C., Eddington, K. M., Levin-Aspenson, H., \& Kwapil, T. R. (2014). Everyday creativity in daily life: An experience-sampling study of "little c" creativity. Psychology of Aesthetics, Creativity, and the Arts, 8(2), 183-188. https://doi.org/10.1037/a0035722

Tanggaard, L. (2015). The creative pathways of everyday life. Journal of Creative Behavior, 49(3), 181-193. https://doi.org/10.1002/jocb.95

van Vuuren, K. (2002). Beyond the studio: A case study of community radio and social capital. Media International Australia, Incorporating Culture \& Policy, 103(1), 94-108. https://doi.org/10.1177/1329878X0210300113

van Vuuren, K. (2004). Community participation in Australian community broadcasting: A comparative study of rural, regional and remote radio. [Doctoral dissertation, Griffith University].

van Vuuren K. (2006). Community broadcasting and the enclosure of the public sphere. Media, Culture $\mathcal{E}$ Society 28(3), 379-392.

Vidal, M. M. (2019). Radio as a sociability space. Journal of Radio \& Audio Media, 26(1), 75-78. https://doi.org/10.1080/19376529.2019.1564995

World Health Organization. (2020). Basic documents: Forty-ninth edition (including amendments adopted up to 31 May 2019). World Health Organization.

3ZZZ. (n.d.). Ethnic Community Broadcasting Association of Victoria Ltd. https://www.3zzz.com.au/ 